\title{
Optimal Value of Series Capacitors for Uniform Field Distribution in Transmission Line MRI Coils
}

\author{
Vitaliy Zhurbenko \\ Technical University of Denmark, 2800 Kongens Lyngby, Denmark \\ Correspondence should be addressed to Vitaliy Zhurbenko; vz@elektro.dtu.dk
}

Received 13 August 2015; Revised 20 October 2015; Accepted 4 November 2015

Academic Editor: Guiyun Tian

Copyright (C) 2016 Vitaliy Zhurbenko. This is an open access article distributed under the Creative Commons Attribution License, which permits unrestricted use, distribution, and reproduction in any medium, provided the original work is properly cited.

\begin{abstract}
Transmission lines are often used as coils in high field magnetic resonance imaging (MRI). Due to the distributed nature of transmission lines, coils based on them produce inhomogeneous field. This work investigates application of series capacitors to improve field homogeneity along the coil. The equations for optimal values of evenly distributed capacitors are derived and expressed in terms of the implemented transmission line parameters. The achieved magnetic field homogeneity is estimated under quasistatic approximation and compared to the regular transmission line resonator. Finally, a more practical case of a microstrip line coil with two series capacitors is considered.
\end{abstract}

\section{Introduction}

Magnetic resonance (MR) imaging at high field strength can potentially offer higher spatial and/or temporal resolution then conventional (low field) MRI systems. These benefits will facilitate a significant increase in diagnostic accuracy for certain medical applications. There are, however, several technological challenges associated with high field operation. One of them is high magnetic resonance frequency, which leads to inhomogeneity of the alternating magnetic field due to the short wavelength of the field. This calls for technological improvements to meet the demands for magnetic field distribution.

The purpose of MR coils is to generate and sense alternating magnetic fields in MR systems. While loop coils are a conventional choice for low field (low frequency) MR systems [1-3], transmission line sections operating in standing wave mode are often used in high field systems [4-6]. The maxima and minima of the standing wave result in inhomogeneous field generated by the coil. Such inhomogeneity leads to degradation of MR image quality.

Several approaches to improve homogeneity have been suggested in the literature. These include implementation of alternating impedance transmission lines $[7,8]$, metamaterial inspired structures $[9,10]$, and substituting coil with several shorter coils with individual feeding [11]. In this work, the approach of inserting series capacitors into transmission line coil is investigated. Series capacitors compensate for the phase shift in self-inductance of the transmission line section. This compensation results in a more uniform current distribution on the line. The uniform current distribution, in its turn, leads to homogeneous magnetic field. The equations for optimal values of evenly distributed series capacitors are derived. The achieved homogeneity of the magnetic field is estimated using Biot-Savart's law and compared to a regular transmission line resonator. An example of a transmission line coil using suspended microstrip technology is considered.

\section{Coil Design for Uniform Current Distribution}

In order to boost the sensitivity of transmission line coils they are usually operated as resonators. Hence, the loading of transmission line coils is either open-circuit, short-circuit, or purely reactive. Such a loading results in infinite standing wave ratio (assuming lossless case) where the distance between the consecutive minima or maxima is one-half a wavelength [12]. To avoid dark spots on MR image and achieve reasonable homogeneity, current minima should be avoided. For that reason, coils based on transmission lines are almost always shorter than half a wavelength and proper 
capacitive loading is used to achieve symmetric current distribution along the line $[7,8,13-15]$. An example of a normalized current distribution $|I(z)|$ on a $25 \mathrm{~cm}$ long transmission line at $298 \mathrm{MHz}$ is shown in Figure 1 (dashed red curve).

The current reduces at the ends of the line due to destructive interference of the incident and reflected waves.

It is, however, preferable to have a uniform current distribution. That would result in a homogeneous magnetic field along the line.

A more uniform current distribution can be achieved by inserting series capacitors $C_{s}$ into the transmission line (Figure 1(b)). These capacitors can compensate for selfinductance of the transmission line by introducing opposite phase shift. The loading capacitor $C_{L}$, in its turn, should be chosen to provide symmetry of the current distribution. This structure (Figure 1(b)) results in a smaller standing wave ratio than in the conventional case (Figure 1(a)). An example of current distribution on a line using two series capacitors is shown in Figure 1(c) (solid blue curve). As can be seen, the resulting uniformity of the current distribution is considerably improved.

Obviously, the higher the number of capacitors, the more uniform the current distribution can be achieved and, consequently, the higher the magnetic field homogeneity will be.

The value of the series capacitor $C_{s}$ should be chosen such that it compensates the phase shift introduced by one section of the transmission line (Appendix B):

$$
C_{s}=\frac{\sin (\beta l / N)}{4 \pi f_{0} Z_{0}(1-\cos (\beta l / N))},
$$

where $l$ is the total length of the transmission line coil; $N$ is the number of transmission line sections after inserting series capacitors (e.g., using two series capacitors will result in $N=$ 3); $\beta$ is the phase constant; $Z_{0}$ is the characteristic impedance of the implemented transmission line; and $f_{0}$ is the operating frequency. A frequency of $298 \mathrm{MHz}$ is used in all examples here, which is close to operating frequency of MR systems for hydrogen imaging with $7 \mathrm{~T}$ magnetic field.

The expression for the loading capacitor, which will result in the optimal current distribution, is derived imposing current symmetry condition for $l / N$ length line (Appendix A):

$$
C_{L}=2 C_{s}=\frac{\sin (\beta l / N)}{2 \pi f_{0} Z_{0}(1-\cos (\beta l / N))} .
$$

For example, for $50 \Omega$ line, the calculated values of the capacitors using (1) and (2) at $f_{0}=298 \mathrm{MHz}, \beta=7.28$, and $N=3$ are $C_{L} \approx 34.2 \mathrm{pF}$ and $C_{s} \approx 17.1 \mathrm{pF}$.

Having the values of the capacitors and knowing the parameters of the transmission line, current distribution can be found using well-developed transmission line analysis [12]. The current on $n$th transmission line section

$$
I_{n}(z)=\frac{V_{0, n}^{+}}{Z_{0}}\left(e^{-j \beta(z+(N-n) l / N)}-\Gamma_{L, n} e^{j \beta(z+(N-n) l / N)}\right),
$$

where $\Gamma_{L, n}$ and $V_{0, n}^{+}$are the load reflection coefficient and amplitude of the incident wave at the end of $n$th transmission

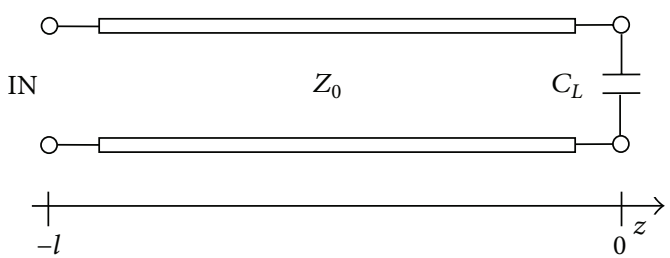

(a)

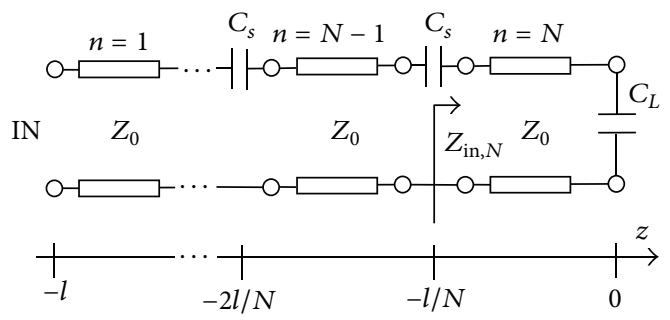

(b)

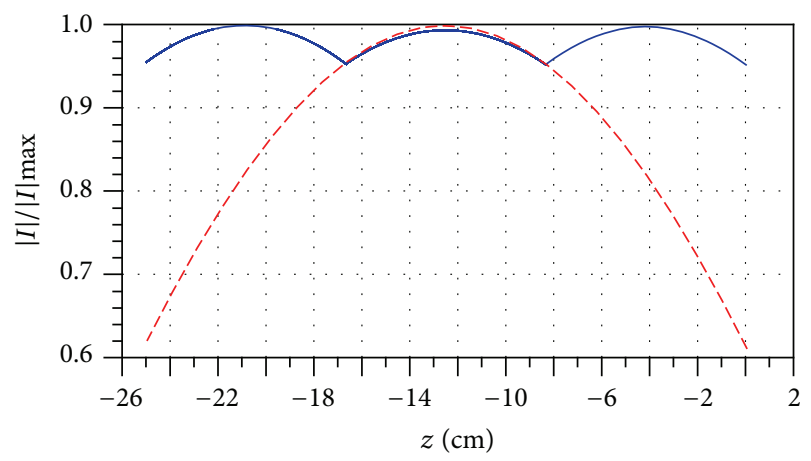

- - - Without series capacitors (Figure 1(a))

W With 2 series capacitors $C_{s}$ (Figure $\left.1(\mathrm{~b})(N=3)\right)$

(c)

FIgURE 1: (a) Regular transmission line resonator. (b) Transmission line coil with series capacitors $C_{s}$. (c) Corresponding normalized magnitude of the current distribution. Here $z$ is the longitudinal coordinate, $l$ is the total length of the coil, and $N$ is the number of sections.

line section, respectively, $n=1 \cdots N$. The total current $I(z)$ is then a combination of currents on all transmission line sections $I_{n}(z)$.

The amplitude of the incident wave at the end of $n$th transmission line section

$$
V_{0, n}^{+}= \begin{cases}\frac{V_{g}}{2} \frac{\left(1-\Gamma_{g}\right) e^{-j \beta l / N}}{1-\Gamma_{g} \Gamma_{L, n} e^{-j 2 \beta l / N}}, & \text { when } n=1, \\ \frac{V_{\mathrm{in}, n}}{e^{j \beta l / N}+\Gamma_{L, n} e^{-j \beta l / N}}, & \text { when } 2 \leq n \leq N .\end{cases}
$$

Here $V_{g}$ is the voltage of the generator, which excites the transmission line coil; $V_{\mathrm{in}, n}$ is the total voltage at the input terminals of $n$th transmission line section. Generator reflection coefficient $\Gamma_{g}=\left(Z_{g}-Z_{0}\right) /\left(Z_{g}+Z_{0}\right)$, where $Z_{g}$ is the impedance of the generator. Reflection coefficient at the end 
of $n$th transmission line section $\Gamma_{L, n}=\left(Z_{L, n}-Z_{0}\right) /\left(Z_{L, n}+Z_{0}\right)$, where

$$
Z_{L, n}= \begin{cases}\frac{1}{j 2 \pi f_{0} C_{L}}, & \text { when } n=N, \\ \frac{1}{j 2 \pi f_{0} C_{s}}+Z_{0} \frac{Z_{L, n+1}+j Z_{0} \tan (\beta l / N)}{Z_{0}+j Z_{L, n+1} \tan (\beta l / N)}, & \text { when } 1 \leq n \leq N-1 .\end{cases}
$$

The field strength due to $I(z)$ can be estimated using BiotSavart law:

$$
|\mathbf{H}(z, r)|=\frac{r}{4 \pi} \int_{-l}^{0} \frac{\left|I\left(z^{\prime}\right)\right| d z^{\prime}}{\left(r^{2}+\left[z^{\prime}-z\right]^{2}\right)^{3 / 2}},
$$

where $r$ is the perpendicular distance to the conductor carrying the current from the point of observation. Since the magnetic flux density is proportional to the magnetic field ( $\mathbf{B}=\mu \mathbf{H}$, where $\mu$ is the permeability), it is possible to estimate the produced B field. Integration in (6) is limited to $-l \cdots 0$ since there is no current outside this region.

Equation (6) is used to estimate the field distributions due to currents in Figure 1(c). This would allow assessing the achieved field homogeneity for the conventional case and the case with series capacitors. The results for $r$ of $3 \mathrm{~cm}$ and $6 \mathrm{~cm}$ are shown in Figure 2.

The presented data indicates that the insertion of series capacitors considerably improves homogeneity of the field. For example, at $r=3 \mathrm{~cm}$, homogeneity, which is defined as a ratio

$$
\frac{\min \{|\mathbf{B}(z)|\}}{\max \{|\mathbf{B}(z)|\}},
$$

increases from $31 \%$ to $49 \%$.

Since the field strength reduces at the ends of the line (Figure 2), a higher degree of homogeneity can be achieved by reducing the field of view (the imaging domain above the coil). For example, limiting the field of view to $z=$ $-5 \mathrm{~cm} \cdots-20 \mathrm{~cm}$ will result in increase in homogeneity from $81 \%$ (Figure 4(a)) to $95 \%$ (Figure 4(b)).

It is important to keep in mind that the presented field distribution in Figure 2 is not for the entire transmission line, but only due to the current distribution $|I(z)|$ in Figure 1. An accurate field distribution for more practical coil realizations should be obtained by full-wave simulations. An example of a microstrip line coil with two distributed series capacitors is considered in the following section.

\section{Example of a Microstrip Line Coil}

The design approach described above is, in general, applicable to any coil based on TEM or quasi-TEM transmission line. A more realistic example of a transmission line coil using suspended microstrip technology is considered in this section. The impedance of the implemented suspended microstrip transmission line is $50 \Omega$. The height (distance between the ground plane and the suspended Printed Circuit Board (PCB)) $h$ is chosen such that the PCB is conveniently supported by the feeding SMA connector and is equal to $4.15 \mathrm{~mm}$. The implemented PCB is based on FR4 laminate sheet with thickness $h_{s}=1.55 \mathrm{~mm}$. The top and side views of the regular $50 \Omega$ line using this technology are shown in Figure 3(a).

The width $W_{1}$ of the $50 \Omega$ line is found using commercially available models for microstrip lines on multilayer substrate and is equal to $22.5 \mathrm{~mm}$. The effective dielectric constant of such a microstrip line is approximately 1.36 . The total length of the coil is $25 \mathrm{~cm}$, which corresponds to electrical length of nearly $0.3 \lambda$.

A transmission line with two series capacitors $C_{s}$ is shown in Figure 3(b). The length of the first and the last transmission line sections $l_{1}$ is one-third of the line in Figure $3(\mathrm{a})$. The conductor of the second section (the one in the middle) is printed on the opposite side of the PCB. In this way, capacitors can be formed by overlapping adjacent transmission line sections. Obviously, the propagation constant is different for the second transmission line section. To compensate for that, the physical length $l_{2}$ had to be slightly increased, such that the electrical lengths (and current distribution) of all three sections are identical. The corresponding parameters of the transmission line are $W_{2}=18.1 \mathrm{~mm}$ and $l_{2}=89.5 \mathrm{~mm}$.

The width of the capacitor plates is chosen the same as the width of the first line $W_{1}$ in order to minimize the discontinuity effect associated with the line-capacitor interconnect.

The length of the capacitor is estimated using expression for the capacitance of flat, parallel metallic plates:

$$
l_{c}=\frac{C_{s} h_{s}}{W_{1} \varepsilon_{0} \varepsilon_{r}}
$$

Substituting $C_{s}=17.1 \mathrm{pF}, h_{s}=1.55 \mathrm{~mm}, W_{1}=22.5 \mathrm{~mm}$, and $\varepsilon_{r}=4.55$ in this equation results in $l_{c} \approx 29.3 \mathrm{~mm}$. Apparently, the length of the capacitor is a significant fraction of the transmission line length; therefore, the capacitor can barely be considered as a lumped capacitor. This will of course have impact on the overall field distribution and its resemblance to the ideal case considered in the previous section.

Equation (1) indicates that the higher number of capacitors (smaller $l / N$ ratio) leads to higher value of $C_{s}$. Should we have chosen $N>3$, it would be difficult to realize larger 


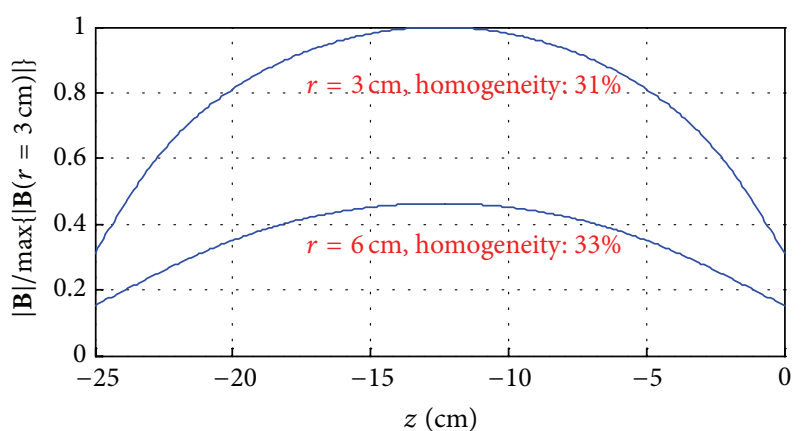

(a)

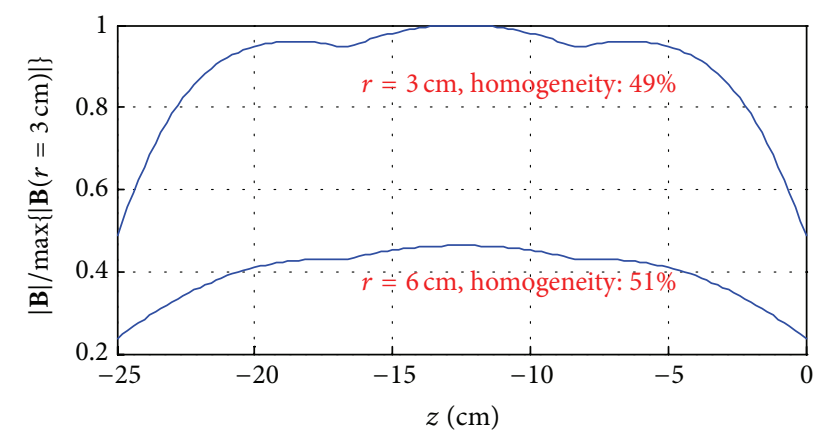

(b)

Figure 2: Normalized magnitude of $\mathbf{B}$ field distribution (a) due to current on a regular transmission line (dashed red curve in Figure 1) and (b) for the case with two series capacitors (solid blue curve in Figure 1).

capacitors in the present configuration and would require implementation of lumped capacitors instead.

Full-wave simulations of microstrip structures in Figure 3 can take into account the distributed behavior of the capacitors, as well as the influence of the ground plane current and the current through the loading capacitor. Initial simulations showed slight asymmetry in current distribution due to fringing effects at the ends of the microstrip lines, nonideal microstrip mode excitation, and current through the loading capacitor (oriented normally to the ground plane). To compensate for that, the value of the loading capacitor $C_{L}$ of the regular transmission line resonator (Figure 3(a)) had to be reduced to $7.2 \mathrm{pF}$ (the initial value calculated from (2) for $N=1$ is $8.3 \mathrm{pF}$ ). The corresponding value for the microstrip line with two series capacitors (Figure 3(b)) $C_{L}=22 \mathrm{pF}$. The value of the series capacitors has not been changed.

The magnetic field profiles for the coils in Figure 3 are illustrated by full-wave simulations in free space using commercial finite integration technique solver [16] (Figure 4). The sinusoidal current distribution in the regular transmission line resonator equates to the $\mathbf{B}$ field distribution in Figure 4(a) with maximum at the center. As it was predicted, a more uniform current profile on the line with series capacitors leads to more uniform field behavior as seen in Figure 4(b).

To evaluate the efficiency of the designed coil in the presence of lossy tissue, phantom studies were conducted. The simulated phantom has a shape of rectangular cuboid

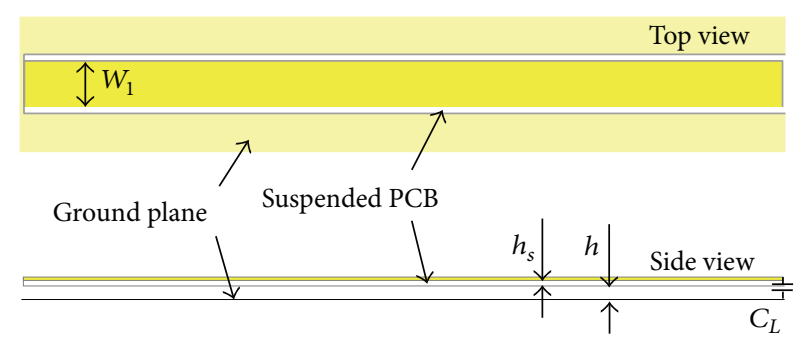

(a)

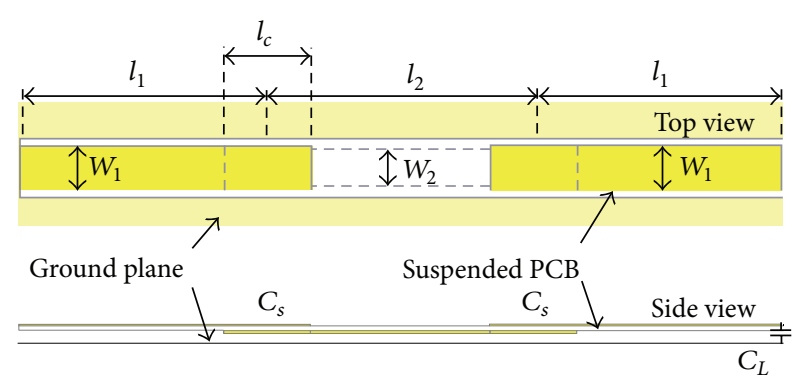

(b)

FIGURE 3: (a) Sketch of a regular suspended microstrip transmission line resonator. (b) Transmission line coil with two series parallel plate capacitors $C_{s}$. Air gap $h=4.15 \mathrm{~mm}, \mathrm{PCB}$ height $h_{s}=1.55 \mathrm{~mm}$, $l_{1}=83.3 \mathrm{~mm}, l_{2}=89.5 \mathrm{~mm}, l_{c}=29.3 \mathrm{~mm}, W_{1}=22.5 \mathrm{~mm}$, and $W_{2}=18.1 \mathrm{~mm}$.

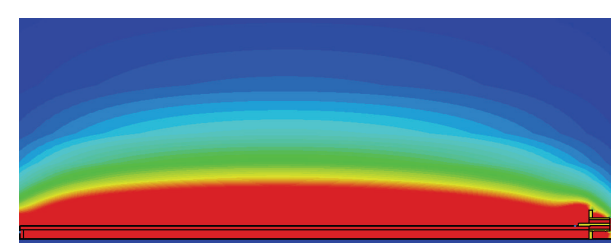

(a)

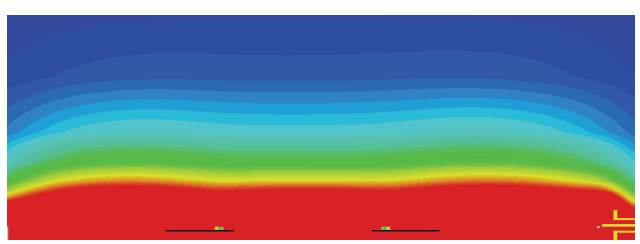

(b)

FIGURE 4: Comparison of the normalized magnitudes of the magnetic field distribution between (a) the regular line and (b) the line with two series parallel plate capacitors in free space.

and placed $5 \mathrm{~mm}$ above the coil. Dielectric properties of the phantom material correspond to average properties of brain at $289 \mathrm{MHz}\left(\varepsilon_{r}^{\prime} \approx 52, \varepsilon_{r}^{\prime \prime} \approx 33\right.$, built-in tissue model in [16]). As can be seen from the simulation results presented in Figure 5, both coils exhibit comparable maximum field generated by a unit of the RF input signal.

The coil with two series capacitors still offers better homogeneity despite exhibiting slight field asymmetry due to distributed nature of losses in the phantom. This asymmetry could be minimized by slightly adjusting the series capacitors when designing for a particular imaging application. 


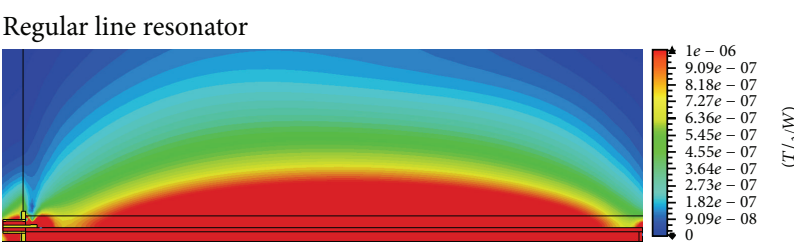

Coil with 2 series capacitors

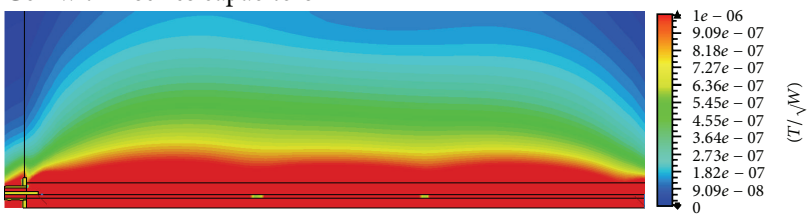

(a)

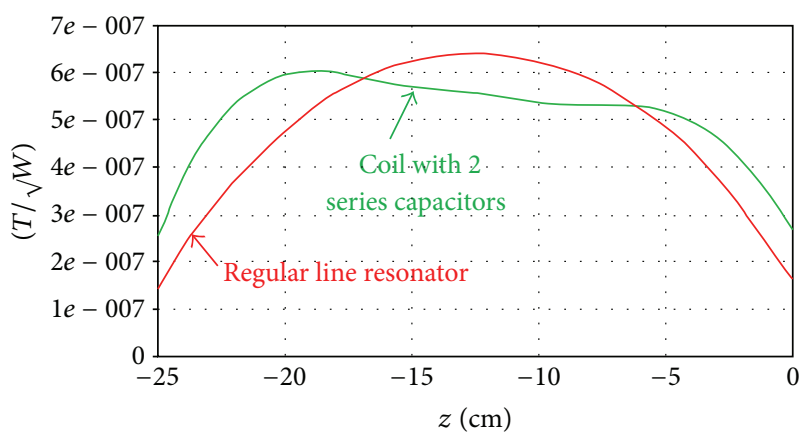

(b)

Figure 5: Simulated $\mathbf{B}_{1}{ }^{+}$field in $[T / \sqrt{ } W]$ generated by a unit of the $\mathrm{RF}$ input signal in presence of a phantom. (a) Slice along the line and (b) comparison of two coils at $r=3 \mathrm{~cm}$.

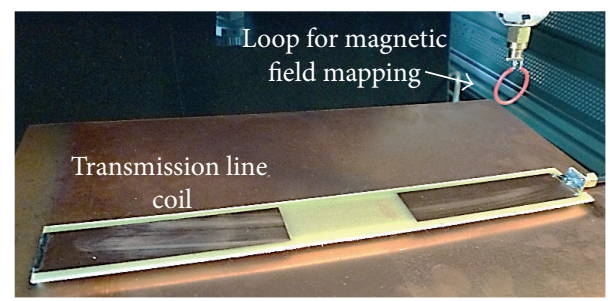

FIGURE 6: Experimental setup for magnetic field mapping.

The coil with series capacitors was fabricated and its field was mapped in free space (Figure 6).

A lumped capacitor was used for $C_{L}$ in the fabricated coil. The coil was tuned to $298 \mathrm{MHz}$ and matched to $50 \Omega$. A small loop (15 $\mathrm{mm}$ in diameter) was implemented for mapping the magnetic field distribution by moving it along the microstrip as illustrated in Figure 6. A vector network analyzer was used to measure coupling between the line and the loop probe. Assuming that the loop is sensitive only to the magnetic field, the measured $\left|S_{21}\right|$ will be proportional to the magnetic field generated by the coil. In this way, the profile of the magnetic field distribution is obtained with $1 \mathrm{~cm}$ step, as illustrated in Figure 7.

The data in Figure 7 presents comparison between the simulated and measured magnetic field distributions $3 \mathrm{~cm}$ and $6 \mathrm{~cm}$ above the transmission line surface. The field

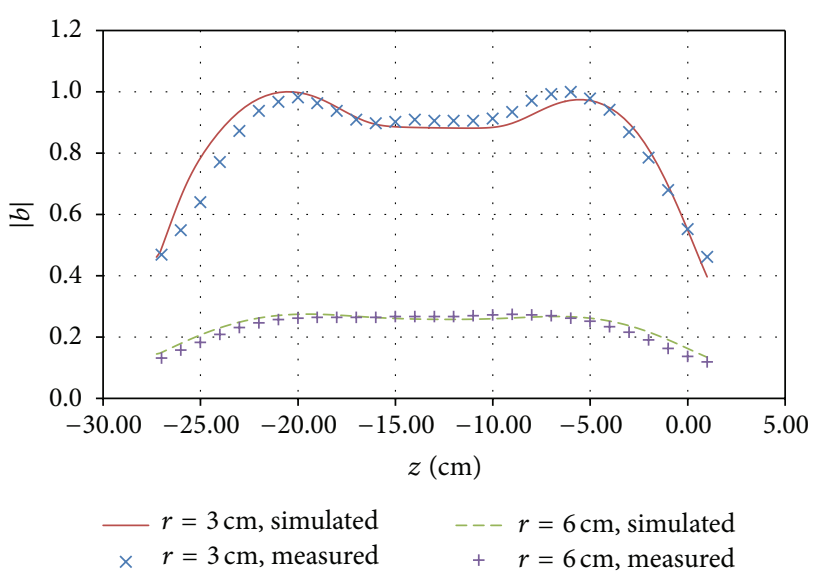

FIGURE 7: Normalized magnitude of the $\mathbf{B}$ field distribution for the microstrip transmission line coil with two series capacitors.

profile exhibits two peaks due to distributed behavior of the implemented series capacitors. The achieved homogeneity of the coil for $r=3 \mathrm{~cm}$ is $55 \%$.

The coil with evenly distributed capacitors has been considered in this work. It is expected to achieve better homogeneity by adjusting the length of particular transmission line sections. This approach, however, has been left out of the scope of this work.

\section{Conclusion}

It was demonstrated that the magnetic field homogeneity for the transmission line MR coils can be improved by inserting series capacitors. The higher the number of capacitors is, the more uniform the field can be generated. Theoretically, any degree of homogeneity can be achieved. The only limitation is the finite length of the coil (which can be overcome by reducing the field of view) and, potentially, losses in the lumped capacitors. Another benefit of using series capacitors is the capability to provide in a simple manner the required uniform field over a very long length and construct transmission line coils, which can be even longer than one-half of a wavelength.

It is shown that the profile of the current distribution can be changed inserting series capacitors. Values for evenly distributed capacitors have been derived in this work (equal value capacitors inserted between equal length line sections). It is expected that even higher homogeneity over a wider field of view could be achieved using unevenly distributed capacitors with variable values.

The approach developed here to control magnetic field distribution would be useful in the design of coils for high field systems for imaging in large in terms of wavelength objects.

The derived equations have been used in the design example of a suspended microstrip coil with two series capacitors. This particular coil realization required slight reduction of the loading capacitor value $C_{L}$ in order to compensate for nonideal behavior and achieve symmetric field distribution. 


\section{Appendices}

\section{A. Deriving Equation for Loading Capacitor}

The current distribution has highest homogeneity when the maximum appears at the center of the transmission line section. Considering the current on the last transmission line section ((3) when $n=N)$ the maximum of the current magnitude will appear at $z=-l / 2 N$ if $e^{j\left(\theta_{L}+2 \beta z\right)}=-1$, where $\theta_{L}$ is the phase of the load reflection coefficient. Consequently, $\theta_{L}-l \beta / N=-\pi$.

Load reflection coefficient in terms of normalized reactance of the load $x_{L}$ is

$$
\Gamma_{L}=\frac{j x_{L}-1}{j x_{L}+1}=\frac{x_{L}^{2}-1}{x_{L}^{2}+1}+j \frac{2 x_{L}}{x_{L}^{2}+1},
$$

where $x_{L}=-1 / 2 \pi f_{0} C_{L} Z_{0}$. Consider

$$
\begin{aligned}
\tan \theta_{L} & =\frac{\operatorname{Im}\left\{\Gamma_{L}\right\}}{\operatorname{Re}\left\{\Gamma_{L}\right\}}=\frac{2 x_{L}}{x_{L}^{2}-1}=-\tan \left(-\frac{\beta l}{N}+\pi\right) \\
& =\tan \left(\frac{\beta l}{N}\right) .
\end{aligned}
$$

Solving this quadratic equation for $x_{L}$,

$$
\begin{aligned}
x_{L} & =\frac{1 \pm \sqrt{1+\tan ^{2}(\beta l / N)}}{\tan (\beta l / N)}=\frac{1 \pm \sec (\beta l / N)}{\tan (\beta l / N)} \\
& =\frac{\cos (\beta l / N) \pm 1}{\sin (\beta l / N)} .
\end{aligned}
$$

We choose solution with "-" since $x_{L}$ is negative, which leads to the following equation for the loading capacitor:

$$
C_{L}=\frac{-\sin (\beta l / N)}{2 \pi f_{0} Z_{0}(\cos (\beta l / N)-1)}
$$

\section{B. Equation for Series Capacitor}

To derive equation for series capacitor $C_{s}$ the last $(N$ th) transmission line section in Figure 1 is considered. The foregoing section should be loaded with $Z_{L}$ in order to achieve current distribution identical to distribution on the last section. This is described by the following equality: $Z_{\mathrm{in}, N}-j / 2 \pi f_{0} C_{s}=Z_{L}$, where

$$
Z_{\text {in, } N}=Z_{0} \frac{Z_{L}+j Z_{0} \tan (\beta l / N)}{Z_{0}+j Z_{L} \tan (\beta l / N)}
$$

is the input impedance of the last ( $N$ th) transmission line section. Using this equation

$$
\begin{aligned}
& \frac{-j}{2 \pi f_{0} C_{s}} \\
& =\frac{Z_{L}\left(Z_{0}+j Z_{L} \tan (\beta l / N)\right)-Z_{0}\left(Z_{L}+j Z_{0} \tan (\beta l / N)\right)}{Z_{0}+j Z_{L} \tan (\beta l / N)} \\
& =\frac{j \tan (\beta l / N)\left(Z_{L}^{2}-Z_{0}^{2}\right)}{Z_{0}+j Z_{L} \tan (\beta l / N)} .
\end{aligned}
$$

Substituting (A.4),

$$
\begin{aligned}
& \frac{-j}{2 \pi f_{0} C_{s}} \\
& =\frac{j \tan (\beta l / N)\left(-Z_{0}^{2}(\cos (\beta l / N)-1)^{2} / \sin ^{2}(\beta l / N)-Z_{0}^{2}\right)}{Z_{0}+j Z_{0}((\cos (\beta l / N)-1) / \sin (\beta l / N)) \tan (\beta l / N)} \\
& =\frac{j Z_{0}\left(-(\cos (\beta l / N)-1)^{2}-\sin ^{2}(\beta l / N)\right)}{\sin (\beta l / N)} \\
& =j Z_{0} \frac{2 \cos (\beta l / N)-2}{\sin (\beta l / N)}=\frac{-j 2}{2 \pi f_{0} C_{L}},
\end{aligned}
$$

from where

$$
C_{s}=\frac{C_{L}}{2}
$$

\section{Conflict of Interests}

The author declares that there is no conflict of interests regarding the publication of this paper.

\section{Acknowledgment}

The author would like to thank Danish National Research Foundation (Grant DNRF124) for partial support of the activities.

\section{References}

[1] J. J. H. Ackerman, T. H. Grove, G. G. Wong, D. G. Gadian, and G. K. Radda, "Mapping of metabolites in whole animals by ${ }^{31} \mathrm{P}$ NMR using surface coils," Nature, vol. 283, no. 5743, pp. 167-170, 1980.

[2] J. T. Vaughan and J. R. Griffiths, Eds., RF Coils for MRI, John Wiley \& Sons, New York, NY, USA, 2012.

[3] D. T. P. Nilsson, J. J. Mohr, and V. Zhurbenko, "Practical aspects of $13 \mathrm{C}$ surface receive coils with active decoupling and tuning circuit," in Proceedings of the 42nd European Microwave Conference (EuMC '12), pp. 65-68, Amsterdam, The Netherlands, November 2012.

[4] J. T. Vaughan, H. P. Hetherington, J. O. Otu, J. W. Pan, and G. M. Pohost, "High frequency volume coils for clinical NMR imaging and spectroscopy," Magnetic Resonance in Medicine, vol. 32, no. 2, pp. 206-218, 1994.

[5] B. A. Baertlein, Ö. Özbay, T. Ibrahim et al., "Theoretical model for an MRI radio frequency resonator," IEEE Transactions on Biomedical Engineering, vol. 47, no. 4, pp. 535-546, 2000.

[6] R. F. Lee, C. R. Westgate, R. G. Weiss, D. C. Newman, and P. A. Bottomley, "Planar strip array (PSA) for MRI," Magnetic Resonance in Medicine, vol. 45, no. 4, pp. 673-683, 2001.

[7] I. A. Elabyad and A. Omar, "An investigation of alternating impedance microstrip transceiver coil arrays for MRI at 7T," in Proceedings of the IEEE MTT-S International Microwave Symposium (IMS '11), pp. 1-4, IEEE, Baltimore, Md, USA, June 2011.

[8] C. E. Akgun, L. Delabarre, H. Yoo et al., "Stepped impedance resonators for high-field magnetic resonance imaging," IEEE Transactions on Biomedical Engineering, vol. 61, no. 2, pp. 327333, 2014. 
[9] A. Rennings, J. Mosig, A. Bahr, C. Caloz, M. E. Ladd, and D. Erni, "A CRLH metamaterial based RF coil element for magnetic resonance imaging at 7 tesla," in Proceedings of the 3rd European Conference on Antennas and Propagation (EuCAP '09), pp. 3231-3234, Berlin, Germany, March 2009.

[10] A. Senn, A. Peter, and J. G. Korvink, "An 8-channel metamaterial T-R coil at 9.4T," in Proceedings of the ISMRM Annual Meeting, p. 1, Montréal, Canada, 2011.

[11] X. Yan, J. O. Pedersen, L. Wei, X. Zhang, and R. Xue, "Multichannel double-row transmission line array for human MR imaging at ultrahigh fields," IEEE Transactions on Biomedical Engineering, vol. 62, no. 6, pp. 1652-1659, 2015.

[12] D. Pozar and D. M. Pozar, "Transmission line theory," in Microwave Engineering, chapter 2, pp. 48-94, Wiley, 4th edition, 2011.

[13] Y. Pang, Z. Xie, D. Xu et al., "A dual-tuned quadrature volume coil with mixed $\lambda / 2$ and $\lambda / 4$ microstrip resonators for multinuclear MRSI at 7 T," Magnetic Resonance Imaging, vol. 30, no. 2, pp. 290-298, 2012.

[14] R. Ludwig, G. Bodgdanov, J. King, A. Allard, and C. F. Ferris, "A dual RF resonator system for high-field functional magnetic resonance imaging of small animals," Journal of Neuroscience Methods, vol. 132, no. 2, pp. 125-135, 2004.

[15] X. Zhang, K. Ugurbil, R. Sainati, and W. Chen, "An invertedmicrostrip resonator for human head proton MR imaging at 7 tesla," IEEE Transactions on Biomedical Engineering, vol. 52, no. 3, pp. 495-504, 2005.

[16] CST Microwave Studio, 2015, https://www.cst.com/. 


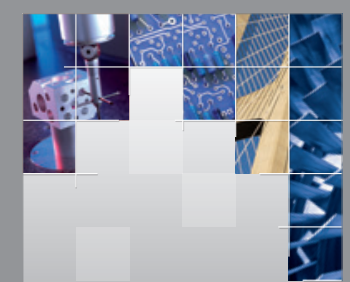

\section{Enfincering}
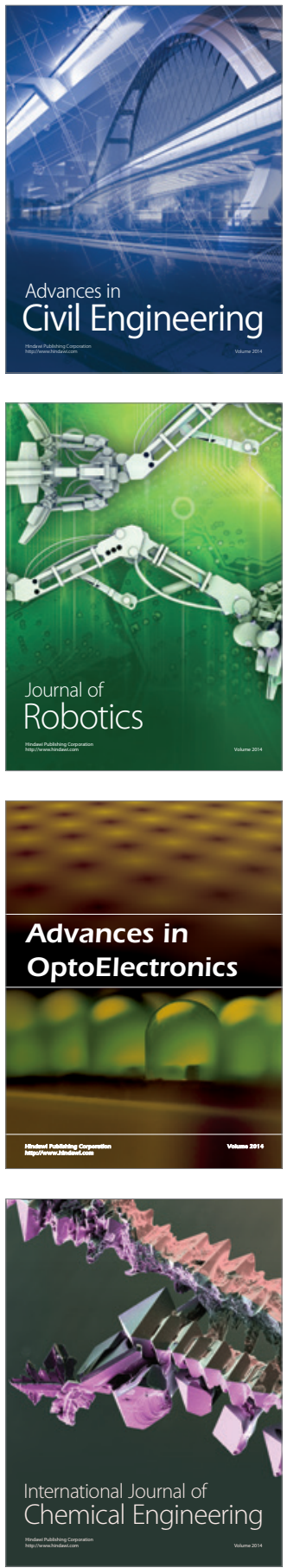

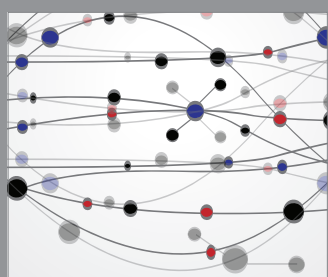

The Scientific World Journal

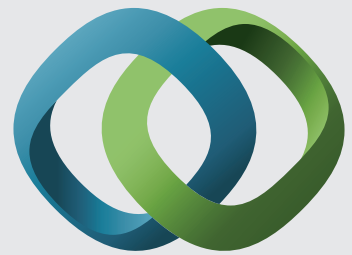

\section{Hindawi}

Submit your manuscripts at

http://www.hindawi.com
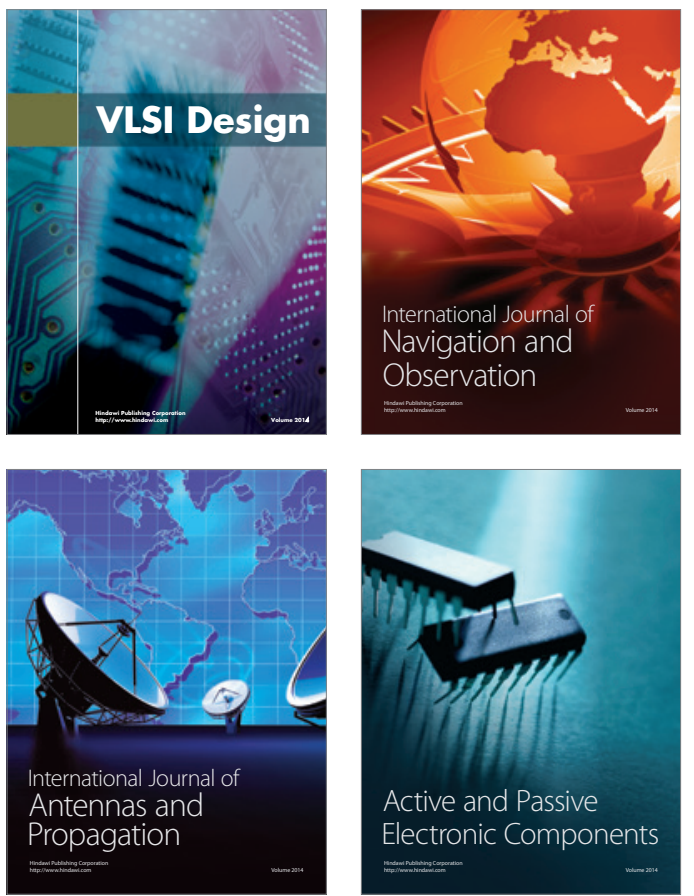
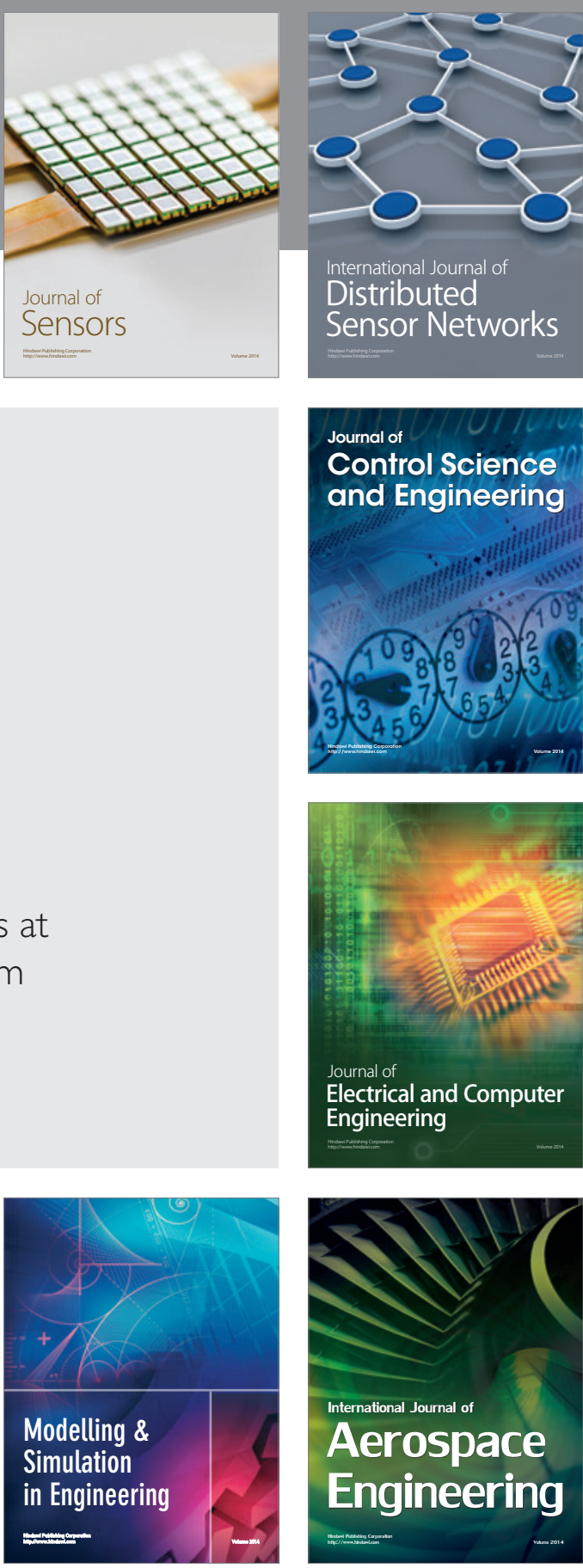

International Journal of

Distributed

Sensor Networks

Journal of

Control Science

and Engineering
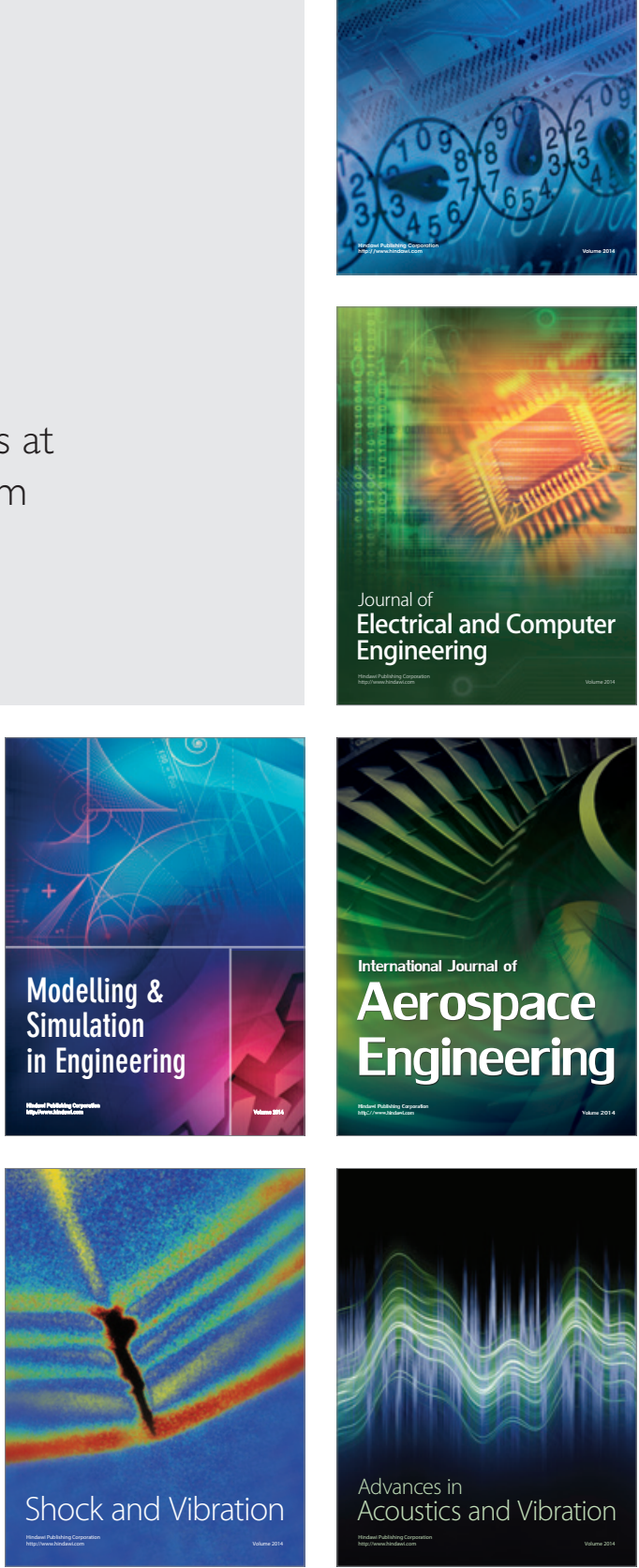